\title{
OPTIMAL RECONFIGURATION OF SUBSTATION TOPOLOGIES IN POWER SYSTEMS FOR ENHANCING NETWORK SECURITY
}

\author{
C. M. QUINTERO ${ }^{1}$, J. M. LÓPEZ-LEZAMA ${ }^{2}$ \& EDWIN RIVAS TRUJILLO ${ }^{3}$ \\ ${ }^{1,2}$ Grupo de Investigación en Manejo Eficiente de la Energía (GIMEL). Departamento de Ingeniería Eléctrica, \\ Universidad de Antioquia, Medellín, Colombia \\ ${ }^{3}$ Grupo de Investigación Interferencia Electromagnética (GCEM), Ingeniería Eléctrica, Facultad de Ingeniería,
} Universidad Distrital Francisco José de Caldas, Bogotá, Colombia

\begin{tabular}{|l|}
\hline ABSTRACT \\
Electric power systems are prone to outages due to natural occurring or intentional phenomena. This paper presents a \\
metaheuristic approach for the optimal reconfiguration of substation topologies under degraded network conditions. The \\
aim of the reconfiguration is the alleviation of violated constraints under single contingencies. The proposed simulated \\
annealing technique performs an intelligent topological exploration of state switches in substations. Such exploration \\
allows finding reconfiguration of substations that would alleviate the consequences of a current outage. In this way, a \\
higher level of security is achieved under N-1 contingencies. The proposed approach considers an AC modeling of the \\
network and a bay-switch model for the assessment of contingencies, guaranteeing realistic conditions of power systems. \\
Several tests are performed on the IEEE-118 bus test system showing the effectiveness of the proposed approach. \\
KEYWORDS: Electric Power Systems, Natural Occurring \& Intentional Phenomena
\end{tabular}

Received: Jun 02, 2020; Accepted: Jun 22, 2020; Published: Oct 10, 2020; Paper Id.: IJMPERDJUN20201497

\section{NOMENCLATURE}

The nomenclature used throughout the paper is provided here for quick reference.

\section{Variables}

$\mathrm{OL}_{\mathrm{i}, 1}$ : $\quad$ Binary variable that takes the value of one if contingency $i$ produces an overload in branch $l$ and zero otherwise.

$\mathrm{OV}_{\mathrm{i}, \mathrm{n}}$ : $\quad$ Binary variable that takes the value of one if contingency $i$ produces a voltage violation at bus $n$.

$\mathrm{CV}_{\mathrm{r}}$ : Binary variable that takes the value of one when there is no power flow convergence for contingency $r$ and zero otherwise.

$\mathrm{DX}_{\mathrm{s}}$ : $\quad$ Binary variable that takes the value of one when a load or generator $s$ is disconnected and zero otherwise.

$\mathrm{P}_{\mathrm{DS}_{\mathrm{n}}}: \quad$ Non served demand at bus $n$.

$\mathrm{P}_{\mathrm{n}}: \quad$ Net active power injection at bus $n$.

$\mathrm{Q}_{\mathrm{n}}$ : $\quad$ Net reactive power injection at bus $n$.

$\mathrm{P}_{1}^{\mathrm{Br}}$ : $\quad$ Active power flow in branch $l$. 
$\mathrm{Q}_{1}^{\mathrm{Br}}: \quad$ Reactive power flow in branch $l$.

$\mathrm{S}_{1}^{\mathrm{Br}}: \quad$ Apparent power flow in branch $l$.

T: $\quad$ Temperature of the Simulated Annealing algorithm.

$O F: \quad$ Objective Function.

\section{Parameters}

$\mathrm{k}_{1}, \mathrm{k}_{2}$ : Penalty constants of the objective function

$\mathrm{P}_{\mathrm{D}_{\mathrm{n}}}: \quad$ Demand at bus $n$.

$\mathrm{G}_{\mathrm{mn}}$ : $\quad$ Real component of the $m, n$ entry of the system admitancematriz.

$\mathrm{B}_{\mathrm{mn}}$ : Imaginary component of the $m, n$ entry of the system admitancematriz.

$\mathrm{V}_{\mathrm{n}}$ : $\quad$ Voltage magnitude at bus $n$.

$\theta_{\mathrm{mn}}: \quad$ Angle between buses $m, n$.

$\mathrm{g}_{\mathrm{mn}}: \quad$ Conductance of the branch connecting nodes $m, n$.

$\mathrm{b}_{\mathrm{mn}}$ : Susceptance of the branch connecting nodes $m, n$

$\mathrm{T}_{\mathrm{o}}$ : Initial temperature of the Simulated Annealing algorithm.

Tfin: $\quad$ Final temperature of the Simulated Annealing algorithm.

nrep: Number of iterations.

dt: $\quad$ Constant of temperature reduction of the Simulated Annealing algorithm.

$\beta$ : $\quad$ Difference between two objective functions of the Simulated Annealing algorithm.

$\theta_{\text {ref }}: \quad$ Reference angle.

$\mathrm{P}_{\mathrm{g}}^{\mathrm{Gen}}$ : Active power generation.

$\mathrm{Q}_{\mathrm{g}}^{\mathrm{Gen}}$ : Reactive power generation.

Sets

Ce: $\quad$ Set of contingencies.

Br: $\quad$ Set of branches.

N: $\quad$ Set of buses.

NCe: Set of contingencies for which there is no convergence of the load flow.

Dcg: Set of loads and generators disconnected due to a contingency. 


\section{INTRODUCTION}

Electric power system operators must guarantee the supply of electrical energy, minimizing operating costs and guaranteeing certain levels of quality, reliability and safety [1],[2]. In this context, the optimization of resources is a priority for system operators. The operation of an electric power system is impacted by a series of factors such as regulatory requirements, climatic phenomena, socio-political events (abnormal conditions of public order) and geological faults that can bring the system to a much higher level of stress. On the other hand, the delay of expansion projects and the continuous growth of demand cause the restrictions to become more acute, increasing the probability of entering operating scenarios that do not allow meeting the reliability and safety criteria required by the regulator [3]. In this regard, several studies have been presented in the specialized literature addressing the topological modification of electric power systems for different purposes, such as overload relief [4], improvement of voltage profile [5], [6], reliability enhancement [7] and minimization of operational costs [8], [9]. In these works, the approach of the solutions is based on the opening of the transmission lines or division of substations. The objective of both solutions is to improve the conditions of the electrical network in terms of safety and economy, compared to those that existed prior to the implementation of the modifications. The aforementioned works do not consider the change of states of all the switches associated with the substations. Other methodologies, such as those presented in [10] and [11] plan the topological changes from the change of state of the switches; However, the substations considered are double-bus and breaker-and-a-half configuration, leaving out the ringtype substations that can be used to reconfigure the system and obtain benefits in terms of safety.

In this work, three types of substations that allow topological reconfigurations in the system are considered. These types are known as breaker-and-a-half, double bus and ring. To solve the proposed optimization model, a Simulated Annealing (SA) metaheuristic was implemented. This method makes an exploration of topologies in a power system through the modification of the states of the switches belonging to the substations with breaker-and-a-half, double bus and ring configurations. The technique is supported by a pre-processing of the information that allows accessing the necessary components of the system in a systematic way to make the modifications efficiently. Similarly, it facilitates the interpretation of the results. The main contribution of this paper, with respect to other works reported in the specialized literature, lies in the search for additional topologies to those that can be found in double bus type substations, and in exploring the characteristics provided by ring and breaker-and-a-half type substations, to improve security conditions in a power system with a degraded network. In this context, a degraded network is understood to be one that is at a point of operation where not all security conditions can be met, either due to network or energy availability. Additionally, the evaluation of the security conditions is done considering an AC model of the network and also considers a bay-switch model for the contingency evaluation. The latter allows an evaluation of the real conditions of release of faults in the power system, since the contingency analysis uses the switches adjacent to the fault point to clear them.

\section{MATHEMATICAL FORMULATION}

The objective function is given by equation (1). It qualifies a topology according to the amount of contingencies that it is able to withstand without causing security violations, considering that demand and generation must remain constant. The first term represents the number of overloads in the event of a contingency. In this case, $O L_{i, l}$ is a binary variable that takes the value of one if contingency $i$ produces an overload on element $l$ and zero otherwise. The second term represents the number of voltage violations for a contingency. In this term the binary variable $O V_{i, n}$ takes the value of one if contingency $i$ produces a voltage violation at bus $n$. The third term is a binary variable that triggers a penalty to the objective function 
due to the non-convergence of the load flow for a given contingency. The binary variable $C V_{r}$ takes the value of one when there is no convergence for contingency $r$. The fourth term of the objective function represents the number of generators or loads that were disconnected as a product of the topological reconfiguration, the binary variable $D X_{S}$ takes the value of one when a load or generator $s$ is disconnected and zero otherwise. The last two terms are multiplied by the penalty factors $k_{1}$ and $k_{2}$.

$$
\text { Minfo }=\sum_{i \in C e} \sum_{l \in B r} O L_{i, l}+\sum_{i \in C e} \sum_{n \in N} O V_{i, n}+k_{1} * \sum_{r \in N C e} C V_{r}+k_{2} * \sum_{s \in D c g} D X_{s}
$$

In all topological variations of the system, it must be ensured that the active and reactive power balance constraints are met. On the other hand, the generation must be kept constant. Constraint (2) indicates that the unserved demand due to load shedding must be less than the node demand. Constraints (3) and (4) indicate the mathematical expressions of the nodal power injections. Equation (5) indicates the active and reactive power components present in the apparent power of the system branches. Equations (6) and (7) are the mathematical expressions of the active and reactive power in the branches of the system, respectively. Equations (8) and (9) indicate the active and reactive power balance constraints per node, respectively. Finally, constraint (10) indicates that the reference angle must be zero.

$$
\begin{aligned}
& 0 \leq\left(P_{D S_{n}}\right) \leq P_{D_{n}}, \forall n \in N \\
& P_{n}=V_{n} \sum_{n} V_{m}\left[G_{m n} \cos \left(\theta_{m n}\right)+B_{m n} \sin \left(\theta_{m n}\right)\right], \forall l \in B r, \forall n \in N \\
& Q_{n}=V_{n} \sum_{n} V_{m}\left[G_{m n} \sin \left(\theta_{m n}\right)-B_{m n} \cos \left(\theta_{m n}\right)\right], \forall l \in B r, \forall n \in N \\
& \left(S_{l}^{B r}\right)^{2}=\left(P_{l}^{B r}\right)^{2}+\left(Q_{l}^{B r}\right)^{2}, \forall l \in B r \\
& P_{l}^{B r}=g_{m n} V_{n}^{2}+g_{m n} V_{m} V_{n} \cos \left(\theta_{m n}\right)-b_{m n} V_{m} V_{n} \sin \left(\theta_{m n}\right), \forall l \in B r \\
& Q_{l}^{B r}=-b_{m n} V_{n}^{2}+g_{m n} V_{m} V_{n} \cos \left(\theta_{m n}\right)-b_{m n} V_{m} V_{n} \sin \left(\theta_{m n}\right), \forall l \in B r \\
& P_{g}^{G e n}-P_{D_{n}}+P_{D S_{n}}=P_{n}, \forall n \in N \\
& Q_{g}^{G e n}-Q_{D_{n}}+Q_{D S_{n}}=Q_{n}, \forall n \in N Q_{g}^{G e n}-Q_{D_{n}}+Q_{D S_{n}}=Q_{n}, \forall n \in N \\
& \theta_{r e f}=0
\end{aligned}
$$

\section{SOLUTION APPROACH}

Simulated Annealing (SA) is a well-known metaheuristic technique inspired in the process of annealing in metallurgy. This metaheuristic has proven to be a successful tool to solve a wide range of optimization problems as reported in [12] and [13]. The implemented SA is illustrated in Fig. 1. In the first stage an initial solution (So) is selected and values are assigned to the algorithm parameters. The initial solution consists of an operating point characterized by the state of the switches. The parameters of the algorithm are the initial temperature $\left(T_{0}\right)$, the initial value of the control variable (T), the final temperature $\left(T_{f i n}\right)$ and the number of iterations $\left(n_{\text {rep }}\right)$. The algorithm uses a temperature reduction function that determines the cooling of the system. In this case, a linear cooling function was used, with a reduction constant $d t$.

In the cooling stage, the SA performs topological explorations according to the selected movement. This is variable and depends on the number of substations that constitute the solution and the number of bays or diameters of the substation that was selected to make changes to the states of its switches. The random movement determines how many and which substations, which bays or diameters and the state changes that will be made to switches during its execution. 
The randomly selected switches and must be assigned new open or closed states, considering the type of substation to which they belong. The objective function is calculated after the application of each movement and its value is compared to that of the best solution found so far (incumbent).

If the value of the current solution is lower, it replaces the current incumbent. When the value of the current solution is greater than that of the incumbent, a random number " $\mu$ " is generated between 0 and 1 . This number is compared with $e^{-\beta / T}$, where $\beta$ is the difference of objective functions and $\mathrm{T}$ is the temperature of the system at which the topology was found. If " $\mu$ " is less than $e^{-\beta / T}$, the new solution is accepted and the algorithm continues with the exploration process. Otherwise, the new solution is rejected and the algorithm continues. The control that is made on the acceptance or rejection of the solutions depends on the temperature. When it is higher, the probability of acceptance of worse solutions increases, giving diversity to the search. On the other hand, when the temperature drops, the acceptance of poor quality solutions decreases, which means that the search around the best solution found is intensified.

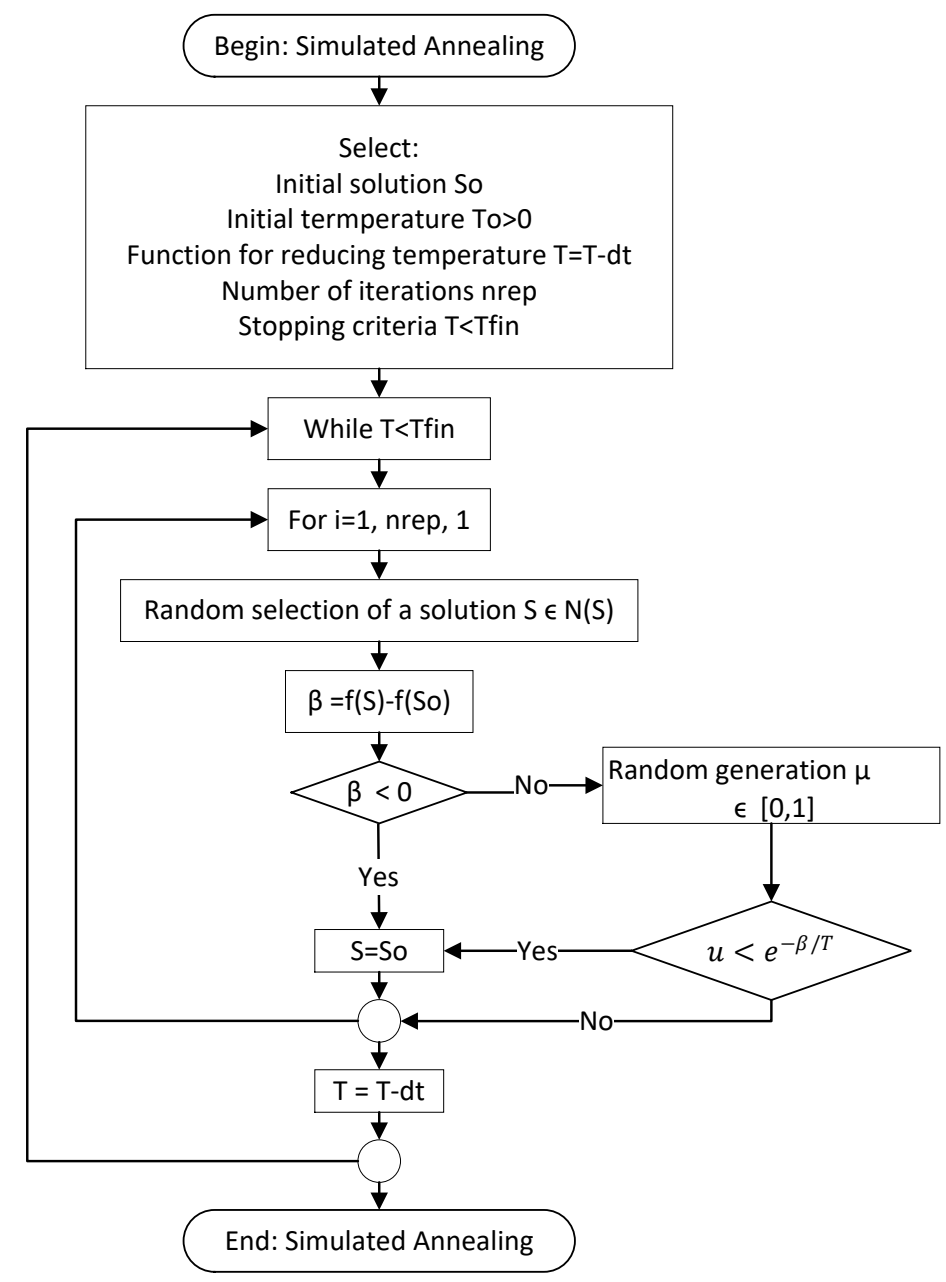

Figure 1: Simulated Annealing Algorithm.

\section{TESTS AND RESULTS}

Several tests were carried out with the IEEE-118 bus test system illustrated in figure2. This system has 184 branches, 91 loads and 19 generators. To perform load flows and contingency analysis, it is necessary to model the system considering the bay - switch characteristics of the components that are connected to each substation. This implies that each substation must be modeled with all the switches that make up each of the connection bays. Figure 3 shows the way in which these 
elements are connected to the different types of substation configurations considered in this work.

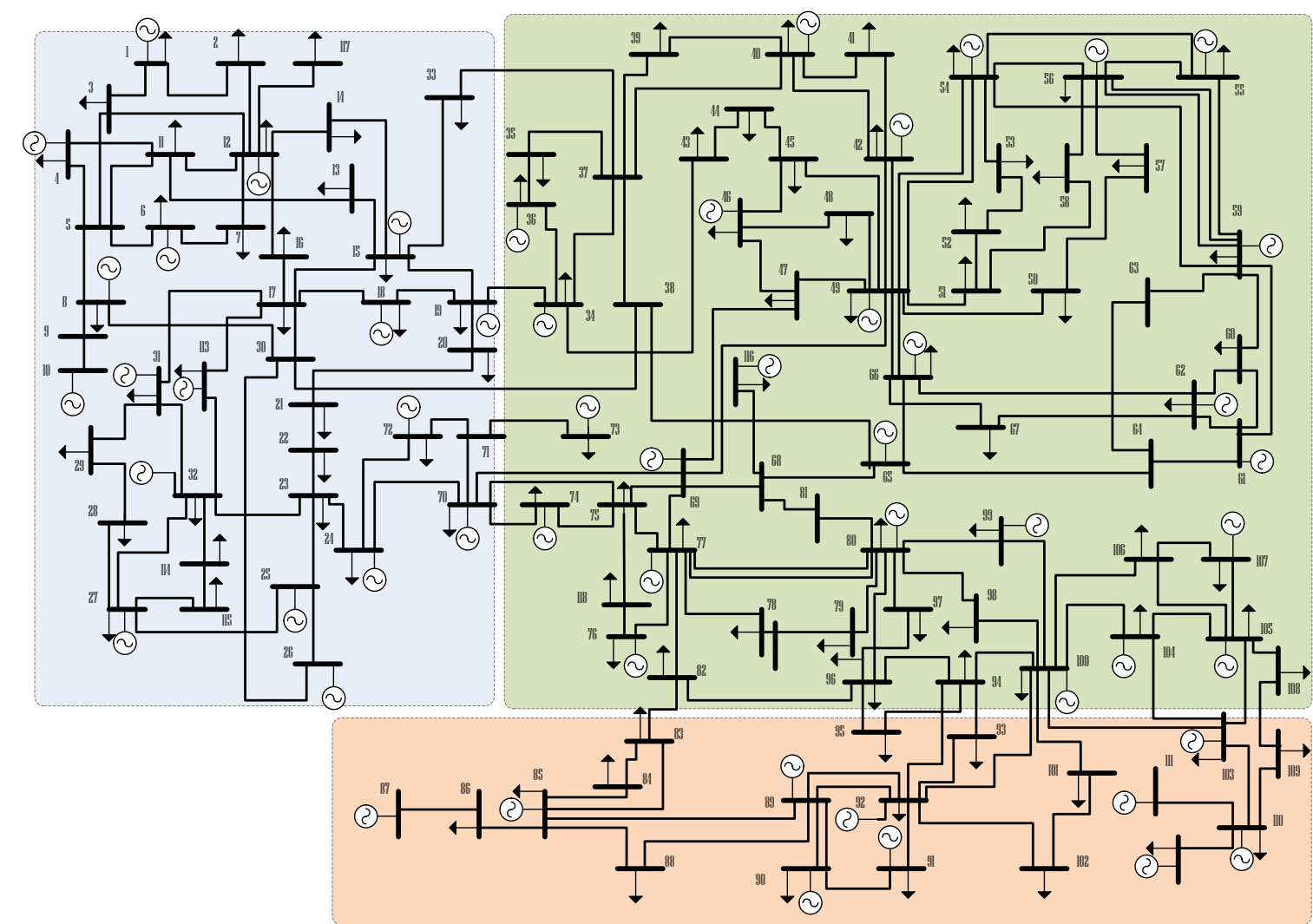

Figure 2: IEEE-118 Bus Test System.

All substations of the IEEE-118 bus test system are of the single bus type. This implies that the connection of each element to the substations is through a single switch. For the execution of the tests it was necessary to carry out a series of transformations to add flexibility to the substations. To this end, a substation configuration type transformation algorithm was developed to transform a single bus type substation to a breaker-and-a-half, double bus or ring type substation. The power system with these modifications was modeled in DigSilent Power Factory.

The substations of nodes $23,47,32,42,66,45,62,51,49,69,54,50,48$ were explored to find topologies that improve the safety of the test system. The thirteen substations are of the breaker-and-a-half type. The contingencies run were of the N-1 type for all branches, except for those transformers with exclusive use of generator connection. This is so that both the generation and the demand remain constant during the tests. The fact that the contingency analysis is performed by clearing the faults with the switches adjacent to the failed element is highlighted. For the base case, it is considered that all substations have their switches closed, which is equivalent to having a single electrical node for each substation. For the base case, the load flow converges for all contingencies; furthermore, all the elements of generation and demand are connected. Therefore, the value of the objective function evaluated in the base is associated with the overloads. The objective function for the base case is 65 , which corresponds to the total number of violations of chargeability and voltage limits. 

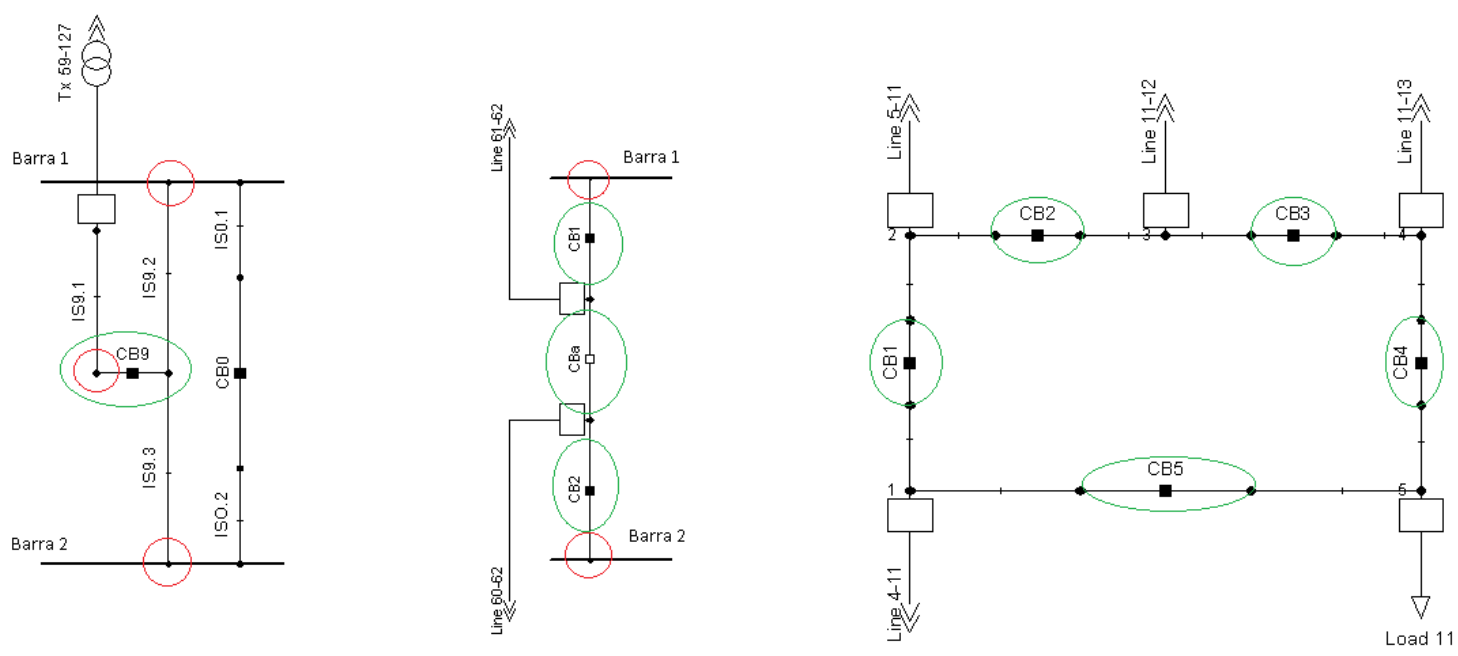

Figure 3: Bay-Switch Model for Different Types of Substations.

The applied SA was tested with 10 simulations combining two parameters: the cooling rate and the number of repetitions. The final and initial temperatures were considered constant with values of 100 and 1 , respectively. Table 1 shows the results for the combination of the mentioned parameters. The reconfigurations found by the SA method were the opening of lines: 31 - 32, 44 - 45, 45 - 49, 46 - 47, 54 - 59 and the attention of the load 54 radially from the transformer 54 - 126. The method found solutions with an objective function value between 57 and 65 . Note that test 7 did not improved the security of the system; however, an improvement of 8 was achieved with test 2 . This means that it is possible to reduce up to 8 constraint violations (chargeability and voltage limits) with the sole reconfiguration of substations.

Table 1: Summary of Results

\begin{tabular}{|c|c|c|c|c|c|}
\hline Test & CoolingRate & Repetitions & ObjectiveFunction & Security Improvement & Time hh:mm:ss \\
\hline 1 & 1 & 2 & 62 & 3 & $0: 07: 59$ \\
\hline 2 & 1 & 5 & 57 & 8 & $0: 21: 03$ \\
\hline 3 & 2 & 2 & 62 & 3 & $0: 04: 22$ \\
\hline 4 & 2 & 5 & 62 & 3 & $0: 10: 42$ \\
\hline 5 & 3 & 2 & 62 & 3 & $0: 03: 05$ \\
\hline 6 & 3 & 5 & 62 & 3 & $0: 07: 55$ \\
\hline 7 & 4 & 2 & 65 & 0 & $0: 02: 25$ \\
\hline 8 & 4 & 5 & 62 & 3 & $0: 05: 46$ \\
\hline 9 & 5 & 2 & 64 & 1 & $0: 01: 55$ \\
\hline 10 & 5 & 5 & 61 & 4 & $0: 04: 41$ \\
\hline
\end{tabular}

\section{CONCLUSIONS}

This paper presented an optimization approach to increase security in power systems through topological modifications. The results with the 118 bus IEEE system evidenced the effectiveness of the proposed algorithm to increase the safety of the system by reconfiguring breaker-and-a-half, double bus and ring substations. The proposed technique can be applied in a whole system or in a zone-by-zone basis.

The bay-switch topology used in this work allows a more realistic evaluation of the impacts of N-1 contingencies. When modeling this topology, for a given contingency two additional elements of the system must be opened. The consideration of the so called bay-switch model is a differentiating factor with respect to other works reported in the technical literature; since the solutions provided by models that do not consider this type of topology must be validated at a later stage by the system operator to verify the real impacts of the topological modifications. 
Regarding the performance of the implemented metaheuristic, it was observed that the execution time the SA is acceptable, ranging from 2 to 20 minutes in a real-size power system. On the other hand, the SA algorithm was able to find up to 8 security improvements (alleviation of chargeability and voltage constraints). In this sense, it would be desirable to test other type of metaheuristics and assess their performance.

\section{ACKNOWLEDGMENTS}

The authors would like to acknowledge the contribution of the Sustainability Project of University of Antioquia for its support in the development of this work.

\section{REFERENCES}

1. D. Urgun, C. Singh, and V. Vittal, "Importance Sampling Using Multilabel Radial Basis Classification for Composite Power System Reliability Evaluation," IEEE Syst. J., vol. 14, no. 2, pp. 2791-2800, Jun. 2020, doi: 10.1109/JSYST.2019.2944131.

2. B. Hu, K. Xie, and H.-M. Tai, "Inverse Problem of Power System Reliability Evaluation: Analytical Model and Solution Method," IEEE Trans. Power Syst., vol. 33, no. 6, pp. 6569-6578, Nov. 2018, doi: 10.1109/TPWRS.2018.2839841.

3. "Reliability Evaluation of Power System Considering Time of Use Electricity Pricing - IEEE Journals \& Magazine." https://ieeexplore.ieee.org/document/8526330 (accessed Aug. 12, 2020).

4. Wei Shao and V. Vittal, "Corrective switching algorithm for relieving overloads and voltage violations," IEEE Trans. Power Syst., vol. 20, no. 4, pp. 1877-1885, Nov. 2005, doi: 10.1109/TPWRS.2005.857931.

5. Y. Bai, H. Zhong, Q. Xia, and C. Kang, "A Two-Level Approach to AC Optimal Transmission Switching With an Accelerating Technique," IEEE Trans. Power Syst., vol. 32, no. 2, pp. 1616-1625, Mar. 2017, doi: 10.1109/TPWRS.2016.2582214.

6. M. Soroush and J. D. Fuller, "Accuracies of Optimal Transmission Switching Heuristics Based on DCOPF and ACOPF," IEEE Trans. Power Syst., vol. 29, no. 2, pp. 924-932, Mar. 2014, doi: 10.1109/TPWRS.2013.2283542.

7. C. Zhang and J. Wang, "Optimal Transmission Switching Considering Probabilistic Reliability," IEEE Trans. Power Syst., vol. 29, no. 2, pp. 974-975, Mar. 2014, doi: 10.1109/TPWRS.2013.2287999.

8. J. D. Fuller, R. Ramasra, and A. Cha, "Fast Heuristics for Transmission-Line Switching," IEEE Trans. Power Syst., vol. 27, no. 3, pp. 1377-1386, Aug. 2012, doi: 10.1109/TPWRS.2012.2186155.

9. M. Khanabadi, H. Ghasemi, and M. Doostizadeh, "Optimal Transmission Switching Considering Voltage Security and N-1 Contingency Analysis," IEEE Trans. Power Syst., vol. 28, no. 1, pp. 542-550, Feb. 2013, doi: 10.1109/TPWRS.2012.2207464.

10. G. Granelli, M. Montagna, F. Zanellini, P. Bresesti, and R. Vailati, "A genetic algorithm-based procedure to optimize system topology against parallel flows," IEEE Trans. Power Syst., vol. 21, no. 1, pp. 333-340, Feb. 2006, doi: 10.1109/TPWRS.2005.860921.

11. A. S. Korad and K. W. Hedman, "Robust Corrective Topology Control for System Reliability," IEEE Trans. Power Syst., vol. 28, no. 4, pp. 4042-4051, Nov. 2013, doi: 10.1109/TPWRS.2013.2267751.

12. J. M. López-Lezama, J. Cortina-Gómez, and N. Muñoz-Galeano, "Assessment of the Electric Grid Interdiction Problem using a nonlinear modeling approach," Electr. Power Syst. Res., vol. 144, no. Supplement C, pp. 243-254, Mar. 2017, doi: 10.1016/j.epsr.2016.12.017.

13. S. Alfonzetti, E. Dilettoso, and N. Salerno, "Simulated annealing with restarts for the optimization of electromagnetic devices, "IEEE Trans. Magn., vol. 42, no. 4, pp. 1115-1118, Apr. 2006, doi: 10.1109/TMAG.2006.871675. 